\title{
Recurrence Relations for Moments of k-th Upper Record Values from Flexible Weibull Distribution and a Characterization
}

\author{
Mahmoud Ali Selim, ${ }^{1, *}$ Hamdy M. Salem² \\ ${ }^{1}$ Department of Statistics, Faculty of Commerce, Al-Azher University, Egypt \& King Khalid University, Community College, Saudi \\ Arabia \\ ${ }^{2}$ Department of Statistics, Faculty of Commerce, Al-Azher University, Egypt \& Qassim University, Community College in Buraidah, \\ Saudi Arabia \\ *Corresponding author: selim.one@gmail.com
}

Received May 10, 2014; Revised May 21, 2014; Accepted May 25, 2014

\begin{abstract}
In this paper, we establish some recurrence relations satisfied by single and product moments of $k$-th upper record values from the flexible Weibull distribution. We also give a characterization of flexible Weibull distribution by using the recurrence relations for single moments.
\end{abstract}

Keywords: order statistics, single moments, product moments, $k$-th upper record values, recurrence relations, flexible Weibull distribution, characterization

Cite This Article: Mahmoud Ali Selim, and Hamdy M. Salem, "Recurrence Relations for Moments of k-th Upper Record Values from Flexible Weibull Distribution and a Characterization.” American Journal of Applied Mathematics and Statistics, vol. 2, no. 3 (2014): 168-171. doi: 10.12691/ajams-2-3-13.

\section{Introduction}

Chandler (1952) was the first to introduce the concept of record values and record statistics. Let $\left\{X_{n}, n \geq 1\right\}$ be a sequence of independent and identically distributed random variables with a cumulative distribution function $\mathrm{F}(\mathrm{x})$ and probability density function $\mathrm{f}(\mathrm{x})$. An observation $\mathrm{X}_{\mathrm{j}}$ will be called an upper record value if its value exceeds all previous observations. Thus, $X_{j}$ is an upper record value if $X_{j}>X_{i}$ for every $i<j$. For a fixed positive integer $k$, Dziubdziela and Kopocinski (1976) defined the sequence $\left\{U_{n}{ }^{(k)}, n \geq 1\right\}$ of $k$-th upper record times for the sequence $\left\{\mathrm{X}_{\mathrm{n}}, \mathrm{n} \geq 1\right\}$ as follows:

$$
\begin{aligned}
& \mathrm{U}_{1}^{(\mathrm{k})}=1, \\
& \mathrm{U}_{\mathrm{n}+1}^{(\mathrm{k})}=\min \left\{\mathrm{j}>\mathrm{U}_{\mathrm{n}}^{(\mathrm{k})}: \mathrm{X}_{\mathrm{j}: \mathrm{j}+\mathrm{k}-1}>\mathrm{X}_{\mathrm{U}_{\mathrm{n}}^{(\mathrm{k})}: \mathrm{U}_{\mathrm{n}}^{(\mathrm{k})}+\mathrm{k}-1}\right\},
\end{aligned}
$$

Where $X_{j: m}$ is the $j$-th order statistic of the sample $X_{1}$, $X_{2}, \ldots, X_{m}$. Then the sequence $\left\{Y_{n}{ }^{(k)}, n \geq 1\right\}$ where $\mathrm{Y}_{\mathrm{n}}{ }^{(\mathrm{k})}=\mathrm{X}_{\mathrm{U}_{\mathrm{n}}}{ }^{(\mathrm{k})}$ is called a sequence of $\mathrm{k}$-th upper record values of $\left\{X_{n}, n \geq 1\right\}$. Note that for $k=1$, we get the usual upper record values as defined in Chandler (1952). For convenience, we also take $\mathrm{Y}_{0}^{(\mathrm{k})}=0$ and $\mathrm{Y}_{1}^{(\mathrm{k})}=\min \left(\mathrm{X}_{1}\right.$, $\left.\mathrm{X}_{2}, \ldots, \mathrm{X}_{\mathrm{N}}\right)$.

Recently, a new two-parameter ageing distribution called a flexible Weibull distribution was proposed by Bebbington et al. (2006) as a generalization of Weibull distribution. The probability density function (pdf) of the flexible Weibull distribution is given by

$$
f(x)=\left(\alpha+\frac{\beta}{x^{2}}\right) e^{\left(\alpha x-\frac{\beta}{x}\right)} e^{-e^{\left(\alpha x-\frac{\beta}{x}\right)}}, x>0, \alpha, \beta \geq 0(1.1)
$$

and the corresponding survival function is

$$
\bar{F}(x)=e^{-e^{\left(\alpha x-\frac{\beta}{x}\right)}}, x>0, \alpha, \beta \geq 0
$$

where $\bar{F}(\mathrm{x})=1-\mathrm{F}(\mathrm{x})$. Note that for $\beta=0$, this distribution reduces to type 1 extreme value distribution, see Johnson et al. (1995). Bebbington et al. (2006) shown that the flexible Weibull distribution is quite flexible, being able to model various ageing classes of lifetime distributions. So we can say that the flexible Weibull distribution is very important in several basic fields include engineering sciences, reliability, biological, demography and actuarial sciences. For more details on the statistical properties and estimation procedures of this distribution, one may refer to Bebbington et al. (2006), Habib et al. (2012) and Singh et al. (2013). Notation $\operatorname{FWD}(\alpha, \beta)$ is used to denote the flexible Weibull distribution with two parameters $\alpha, \beta$.

The moments of $k$-th record values have received considerable attention in the recent years. Many authors derived the recurrence relations for $k$-th record values for different distributions; See, Pawlas and Szynal (1999, 2000), Saran and Singh (2008), Bieniek and Szynal (2002, 2007, 2013), Nain (2010), Selim(2011), Kumar (2011, 2012), Kumar and Khan (2012) and Kumar and Kulshrestha (2013). The recurrence relations for moments of $k$-th record values from flexible Weibull distribution 
have not been considered in the earlier literature. In Section 2, some recurrence relations for the single and product moments of $\mathrm{k}$-th upper record values from the flexible Weibull distribution are derived. In Section 3, a characterization of the flexible Weibull distribution is obtained by using a recurrence relation for single moments. Finally, conclusions are given in Section 4.

We shall denote

$$
\begin{aligned}
& \mu_{n: k}^{(r)}=E\left[\left(Y_{n}^{(k)}\right)^{r}\right], r, n=1,2, \ldots \\
& \mu_{m, n: k}^{(r, s)}=E\left[\left(Y_{m}^{(k)}\right)^{r}\left(Y_{n}^{(k)}\right)^{s}\right], 1 \leq m \leq n-1, r, s=1,2, \ldots \\
& \mu_{m, n: k}^{(r, 0)}=E\left[\left(Y_{m}^{(k)}\right)^{r}\left(Y_{n}^{(k)}\right)^{0}\right]=\mu_{m: k}^{(r)}, 1 \leq m \leq n-1, r=1,2, \ldots \\
& \mu_{m, n: k}^{(0, s)}=E\left[\left(Y_{m}^{(k)}\right)^{0}\left(Y_{n}^{(k)}\right)^{s}\right]=\mu_{n: k}^{(s)}, 1 \leq m \leq n-1, s=1,2, \ldots
\end{aligned}
$$

\section{Recurrence Relations for Single and Product Moments}

The relation between pdf and survival function of $F W D(\alpha, \beta)$ in (1.1) and (1.2), respectively, can be written in the form

$$
f(x)=\left(\alpha+\beta / x^{2}\right)[-\ln \bar{F}(x)] \bar{F}(x)
$$

This relation will be used in this paper to derive some recurrence relations for the single and product moments of $k$-th upper record values from $F W D(\alpha, \beta)$.

Let $\left\{\mathrm{Y}_{\mathrm{n}}{ }^{(\mathrm{k})}, \mathrm{n} \geq 1\right\}$, where $\mathrm{Y}_{\mathrm{n}}{ }^{(\mathrm{k})}=\mathrm{X}_{\mathrm{U}_{\mathrm{n}}}{ }^{(\mathrm{k})}$ be a sequence of $k$-th upper record values arising from $F W D(\alpha, \beta)$. Then the pdf of $\mathrm{Y}_{\mathrm{n}}{ }^{(\mathrm{k})}, \mathrm{n} \geq 1$ (see Dziubdziela and Kopocinski, (1976)) is

$$
\begin{aligned}
& f_{Y_{n}(k)}(x)=\frac{k^{n}}{(n-1) !}[-\ln \bar{F}(x)]^{n-1}[\bar{F}(x)]^{k-1} f(x), \\
& -\infty<x<\infty
\end{aligned}
$$

and the joint pdf of $\mathrm{Y}_{\mathrm{m}}{ }^{(\mathrm{k})}$ and $\mathrm{Y}_{\mathrm{n}}{ }^{(\mathrm{k})}, 1 \leq \mathrm{m}<\mathrm{n}, \mathrm{n} \geq 2$ is

$$
\begin{aligned}
& f_{Y_{m}^{(k)}, Y_{n}^{(k)}}(x, y) \\
& =\frac{k^{n}}{(m-1) !(n-m-1) !}[-\ln \bar{F}(y)+\ln \bar{F}(x)]^{n-m-1}(2.3) \\
& \times[-\ln \bar{F}(x)]^{m-1} \frac{f(x)}{\bar{F}(x)}[\bar{F}(y)]^{k-1} f(y), x<y
\end{aligned}
$$

Now, the recurrence relations for single and product moments of $k$-th upper record values from the flexible Weibull distribution can be introduced in the following theorems.

\section{Theorem 1}

For $n \geq 1$ and $r=2,3, \ldots$,

$$
\begin{aligned}
& \mu_{n: k}^{(r)}=\frac{n \alpha}{(r+1)}\left(\mu_{n+1: k}^{(r+1)}-\mu_{n: k}^{(r+1)}\right) \\
& +\frac{n \beta}{(r-1)}\left(\mu_{n+1: k}^{(r-1)}-\mu_{n: k}^{(r-1)}\right)
\end{aligned}
$$

and for $n=1$

$$
\mu_{1: k}^{(r)}=\frac{\alpha}{(r+1)}\left(\mu_{2: k}^{(r+1)}-\mu_{1: k}^{(r+1)}\right)+\frac{\beta}{(r-1)}\left(\mu_{2: k}^{(r-1)}-\mu_{1: k}^{(r-1)}\right)
$$

\section{Proof}

For $n \geq 2$ and $r=0,1,2, \ldots$, . Using the $p d f$ of $X_{U(n)}$ given in (2.2) and the relation in (2.1), we have

$$
\mu_{n: k}^{(r)}=\frac{k^{n}}{(n-1) !}\left\{\begin{array}{c}
\alpha \int_{0}^{\infty} x^{r}[-\ln \bar{F}(x)]^{n}[\bar{F}(x)]^{k} d x \\
+\beta \int_{0}^{\infty} x^{r-2}[-\ln \bar{F}(x)]^{n}[\bar{F}(x)]^{k} d x
\end{array}\right\}
$$

Integrating by parts treating $[-\ln \bar{F}(\mathrm{x})]^{\mathrm{n}}[\bar{F}(\mathrm{x})]^{\mathrm{k}}$ for differentiation and the rest of the integrand for integration, we obtain

$$
\begin{aligned}
& \mu_{n: k}^{(r)}=\frac{\alpha k^{n}}{(n-1) !(r+1)} \\
& \left\{\begin{array}{c}
k \int_{0}^{\infty} x^{r+1}[-\ln \bar{F}(x)]^{n}[\bar{F}(x)]^{k-1} f(x) d x \\
-n \int_{0}^{\infty} x^{r+1}[-\ln \bar{F}(x)]^{n-1}[\bar{F}(x)]^{k-1} f(x) d x
\end{array}\right\} \\
& +\frac{\beta k^{n}}{(n-1) !(r-1)} \\
& \left\{\begin{array}{c}
k \int_{0}^{\infty} x^{r-1}[-\ln \bar{F}(x)]^{n}[\bar{F}(x)]^{k-1} f(x) d x \\
-n \int_{0}^{\infty} x^{r-1}[-\ln \bar{F}(x)]^{n-1}[\bar{F}(x)]^{k-1} f(x) d x
\end{array}\right\}
\end{aligned}
$$

Upon rewriting the above expression, we immediately obtain the relation (2.4).

Also, the relation (2.5) follows from (2.7) by setting $n=1$.

\section{Remarks 1}

(a) Putting $k=1$, in (2.4) and (2.5) we can deduce the recurrence relations for single moments of the usual upper record values from the flexible Weibull distribution.

(b) Putting $\beta=0$ in (2.4) and simplifying, we get the recurrence relation for single moments of k-th upper record values from type 1 extreme value distribution as follow

$$
\mu_{\mathrm{n}: \mathrm{k}}^{(\mathrm{r})}=\frac{\mathrm{n} \alpha}{(\mathrm{r}+1)}\left(\mu_{\mathrm{n}+1: \mathrm{k}}^{(\mathrm{r}+1)}-\mu_{\mathrm{n}: \mathrm{k}}^{(\mathrm{r}+1)}\right)
$$

\section{Theorem 2}

For $1 \leq m \leq n-2$ and $r, s=0,1,2, \ldots$,

$$
\begin{aligned}
& \mu_{m, n: k}^{(r, s)}=\frac{\alpha m}{(r+1)}\left(\mu_{m+1, n: k}^{(r+1, s)}-\mu_{m, n: k}^{(r+1, s)}\right) \\
& +\frac{\beta m}{(r-1)}\left(\mu_{m+1, n: k}^{(r-1, s)}-\mu_{m, n: k}^{(r-1, s)}\right)
\end{aligned}
$$

and for $\mathrm{m} \geq 1$ and $\mathrm{r}, \mathrm{s}=0,1,2, \ldots$, 


$$
\begin{aligned}
& \mu_{m, m+1: k}^{(r, s)}=\frac{\alpha m}{(r+1)}\left(\mu_{m+1: k}^{(r+s+1)}-\mu_{m, m+1: k}^{(r+1, s)}\right) \\
& +\frac{\beta m}{(r-1)}\left(\mu_{m+1: k}^{(r+s-1)}-\mu_{m, m+1: k}^{(r-1, s)}\right)
\end{aligned}
$$

\section{Proof}

For $1 \leq m \leq n-1$, and $r, s=0,1,2, \ldots$ using (2.3) and (2.1), we have

$$
\begin{aligned}
& \mu_{m, n: k}^{(r, s)}= \\
& \frac{k^{n}}{(m-1) !(n-m-1) !} \int_{0}^{\infty} y^{s} I(y)[\bar{F}(y)]^{k-1} f(y) d y
\end{aligned}
$$

where

$$
\begin{aligned}
& I(y)= \\
& \alpha \int_{0}^{y} x^{r}[-\ln \bar{F}(x)]^{m}[-\ln \bar{F}(y)+\ln \bar{F}(x)]^{n-m-1} d x \\
& +\beta \int_{0}^{y} x^{r-2}[-\ln \bar{F}(x)]^{m}[-\ln \bar{F}(y)+\ln \bar{F}(x)]^{n-m-1} d x
\end{aligned}
$$

Integrating $\mathrm{I}(\mathrm{y})$ by parts, treating $\mathrm{x}^{\mathrm{r}}$ for integration and the rest of the integrand for differentiation in the first term and in the second term treating $\mathrm{x}^{\mathrm{r}-2}$ for integration and the rest of the integrand for differentiation, we obtain

$$
\begin{aligned}
& I(y)=\frac{\alpha}{(r+1)}\left\{\begin{array}{l}
(n-m-1) \int_{0}^{y} x^{r+1}[-\ln \bar{F}(x)]^{m} \\
{[-\ln \bar{F}(y)+\ln \bar{F}(x)]^{n-m-2} \frac{f(x)}{\bar{F}(x)} d x} \\
-m \int_{0}^{y} x^{r+1}[-\ln \bar{F}(x)]^{m-1} \\
{[-\ln \bar{F}(y)+\ln \bar{F}(x)]^{n-m-1} \frac{f(x)}{\bar{F}(x)} d x}
\end{array}\right\} \\
& +\frac{\beta}{(r-1)}\left\{\begin{array}{l}
(n-m-1) \int_{0}^{y} x^{r-1}[-\ln \bar{F}(x)]^{m} \\
{[-\ln \bar{F}(y)+\ln \bar{F}(x)]^{n-m-2} \frac{f(x)}{\bar{F}(x)} d x} \\
-m \int_{0}^{y} x^{r-1}[-\ln \bar{F}(x)]^{m-1} \\
{[-\ln \bar{F}(y)+\ln \bar{F}(x)]^{n-m-1} \frac{f(x)}{\bar{F}(x)} d x}
\end{array}\right\}
\end{aligned}
$$

Substituting the above expression in to (2.11) and simplifying, it leads to equation (2.9). And when $n=m+1$, then

$$
\mu_{m, n: k}^{(r, s)}=\frac{k^{n}}{(m-1) !} \int_{0}^{\infty} y^{s} I(y)[\bar{F}(y)]^{k-1} f(y) d y
$$

Where

$$
I(y)=\alpha \int_{0}^{y} x^{r}[-\ln \bar{F}(x)]^{m} d x+\beta \int_{0}^{y} x^{r-2}[-\ln \bar{F}(x)]^{m} d x
$$

Integrating $I(y)$ by parts, treating $[-\ln \bar{F}(x)]^{m}$ for differentiation and the rest of the integrand for integration we get

$$
\begin{aligned}
& I(y)=\frac{\alpha}{(r+1)}\left\{\begin{array}{l}
y^{r+1}[-\ln \bar{F}(x)]^{m} \\
-m \int_{0}^{y} x^{r+1}[-\ln \bar{F}(x)]^{m-1} \frac{f(x)}{\bar{F}(x)} d x
\end{array}\right\} \\
& +\frac{\beta}{(r-1)}\left\{\begin{array}{l}
y^{r-1}[-\ln \bar{F}(y)]^{m} \\
-m \int_{0}^{y} x^{r-1}[-\ln \bar{F}(x)]^{m-1} \frac{f(x)}{\bar{F}(x)} d x
\end{array}\right\}
\end{aligned}
$$

Substituting the above expression in to (2.12) and simplifying, we obtain (2.10).

\section{Remarks 2}

(a) Putting $\mathrm{k}=1$, in (2.9) and (2.10) we deduce the recurrence relations for product moments of usual upper record values from the flexible Weibull distribution.

(b) Putting $\beta=0$, in (2.9) and simplifying, we get the recurrence relation for product moments of $k$-th upper record values from type 1 extreme value distribution as follow

$$
\mu_{\mathrm{m}, \mathrm{n}: \mathrm{k}}^{(\mathrm{r}, \mathrm{s})}=\frac{\alpha \mathrm{m}}{(\mathrm{r}+1)}\left(\mu_{\mathrm{m}+1, \mathrm{n}: \mathrm{k}}^{(\mathrm{r}+1, \mathrm{~s})}-\mu_{\mathrm{m}, \mathrm{n}: \mathrm{k}}^{(\mathrm{r}+1, \mathrm{~s})}\right)
$$

\section{A Characterization of the Flexible Weibull Distribution}

In this section, we present a characterization for flexible Weibull distribution using the relation in (2.4) based on the following result of Lin(1986).

\section{Proposition 1}

Let $\mathrm{n}_{0}$ be any fixed non-negative integer and let a,b be real numbers such that $-\infty<a<b<\infty$. Let $\mathrm{g}(\mathrm{x}) \geq 0$ be an absolutely continuous function with $\mathrm{g}^{\prime}(\mathrm{x}) \neq 0$ almost everywhere on $(a, b)$. Then the sequence of functions $\left\{[g(x)]^{n} e^{-g(x)}, n \geq n_{0}\right\}$ is complete in L(a,b)if and only if $\mathrm{g}(\mathrm{x})$ is strictly monotone on $(\mathrm{a}, \mathrm{b})$.

\section{Theorem 3}

For a fixed positive integer k. A necessary and sufficient condition for a random variable $\mathrm{X}$ to be distributed with probability density function of the flexible Weibull distribution given by (1.1) is that

$$
\begin{aligned}
& \mu_{n: k}^{(r)}=\frac{n \alpha}{(r+1)}\left(\mu_{n+1: k}^{(r+1)}-\mu_{n: k}^{(r+1)}\right) \\
& +\frac{n \beta}{(r-1)}\left(\mu_{n+1: k}^{(r-1)}-\mu_{n: k}^{(r-1)}\right)
\end{aligned}
$$

\section{Proof}

The necessary part follows immediately from (2.4), on the other hand if the recurrence relation in (3.1) is satisfied, then on rearranging the terms in (3.1) and using (2.2), we have 


$$
\begin{aligned}
& \frac{\alpha k^{n+1}}{(n-1) !(r+1)} \int_{0}^{\infty} x^{r+1}[-\ln \bar{F}(x)]^{n}[\bar{F}(x)]^{k-1} f(x) d x \\
& =\frac{k^{n}}{(n-1) !} \int_{0}^{\infty} x^{r}[-\ln \bar{F}(x)]^{n-1}[\bar{F}(x)]^{k-1} f(x) d x \\
& -\frac{\beta k^{n+1}}{(n-1) !(r-1)} \int_{0}^{\infty} x^{r-1}[-\ln \bar{F}(x)]^{n}[\bar{F}(x)]^{k-1} f(x) d x \\
& +\frac{n \beta k^{n}}{(n-1) !(r-1)} \int_{0}^{\infty} x^{r-1}[-\ln \bar{F}(x)]^{n-1}[\bar{F}(x)]^{k-1} f(x) d x \\
& +\frac{n \alpha k^{n}}{(n-1) !(r+1)} \int_{0}^{\infty} x^{r+1}[-\ln \bar{F}(x)]^{n-1}[\bar{F}(x)]^{k-1} f(x) d x
\end{aligned}
$$

Integrating the last two integrals on the right hand side of (3.2) by parts, we get

$$
\begin{aligned}
& \frac{\alpha k^{n+1}}{(n-1) !(r+1)} \int_{0}^{\infty} x^{r+1}[-\ln \bar{F}(x)]^{n}[\bar{F}(x)]^{k-1} f(x) d x= \\
& \frac{k^{n}}{(n-1) !} \int_{0}^{\infty} x^{r}[-\ln \bar{F}(x)]^{n-1}[\bar{F}(x)]^{k-1} f(x) d x \\
& -\frac{\beta k^{n+1}}{(n-1) !(r-1)} \int_{0}^{\infty} x^{r-1}[-\ln \bar{F}(x)]^{n}[\bar{F}(x)]^{k-1} f(x) d x \\
& -\frac{\beta k^{n}}{(n-1) !} \int_{0}^{\infty} x^{r-2}[-\ln \bar{F}(x)]^{n}[\bar{F}(x)]^{k} d x \\
& +\frac{\beta k^{n+1}}{(n-1) !(r-1)} \int_{0}^{\infty} x^{r-1}[-\ln \bar{F}(x)]^{n}[\bar{F}(x)]^{k-1} f(x) d x \\
& -\frac{\alpha k^{n}}{(n-1) !} \int_{0}^{\infty} x^{r}[-\ln \bar{F}(x)]^{n}[\bar{F}(x)]^{k} d x \\
& +\frac{\alpha k^{n+1}}{(n-1) !(r+1)} \int_{0}^{\infty} x^{r+1}[-\ln \bar{F}(x)]^{n}[\bar{F}(x)]^{k-1} f(x) d x
\end{aligned}
$$

Upon simplification the above expression, we obtain

$$
\begin{aligned}
& \frac{k^{n}}{(n-1) !} \int_{0}^{\infty} x^{r}[-\ln \bar{F}(x)]^{n-1}[\bar{F}(x)]^{k-1} \\
& \left\{f(x)-\left[\left(\alpha+\frac{\beta}{x^{2}}\right)[-\ln \bar{F}(x)] \bar{F}(x)\right]\right\} d x=0
\end{aligned}
$$

Using the proposed 1, we conclude that

$$
f(x)=\left[\left(\alpha+\frac{\beta}{x^{2}}\right)[-\ln \bar{F}(x)] \bar{F}(x)\right]
$$

This proves that $f(x)$ is probability density function of the flexible Weibull distribution.

\section{Conclusions Remarks}

(1). In this paper, some recurrence relations for single and product moments of $\mathrm{k}$-th upper record values from flexible Weibull distribution have been derived.

(2). The recurrence relation for single moments of k-th upper record values has been utilized to obtain a characterization of flexible Weibull distribution.

(3). The recurrence relations for moments of k-records are important because they can be helpful in reducing the amount of direct calculations needed to calculate the moments. And they can be used in a simple recursive manner to express the unknown higher order moments in terms of lower order moments thus making the evaluation of higher moments easy. Also they can be used to characterize the distributions.

\section{References}

[1] Bebbington, M., Lai, C.D. and Zitikis, R. (2006). A flexible Weibull extension, Reliability Engineering and System Safety, 92, 719-726.

[2] Bieniek, M., and Szynal, D. (2002). Recurrence relations for distribution functions and moments of k-th Record values, Journal of Mathematical Sciences, 111, 3511-3519.

[3] Bieniek, M., and Szynal, D. (2007). On k-th record times, record values and their moments, Journal of Statistical Planning and Inference, 137, 12-22.

[4] Bieniek, M., and Szynal, D. (2013). On moments of k-th record values from the linear exponential distribution. Journal of mathematical sciences, 191 (4), 526-537.

[5] Chandler, K.N. (1952). The distribution and frequency of record values, Journal of Royal Statistical Society, B, 14, 220-228.

[6] Dziubdziela, W., and Kopocinski, B. (1976). Limiting properties of the $k$-th record value, Zastosowania Mathematyki, 15, 187-190.

[7] Habib, M.E., Shams, T.M., Selim, M.A. and Hussen, A.A. (2012) Maximum likelihood estimation of the flexible Weibull distribution parameters based on type I censored data. Academy of Business Journal, Al-Azhar University, 9, 1, pp 50-70.

[8] Johnson, N. L., Kotz, S., and Balakrishan, N. (1995). Continuous Univariate Distributions (2nd ed., Vol. 2). New York: Wiley.

[9] Kumar, D. (2011). Recurrence relations for moments of $k$-th lower record values from exponentiated log-logistic distribution and a characterization, International Journal of Mathematical Archive, 2, 6, 813-319.

[10] Kumar, D. (2012): Recurrence relations for marginal and joint moment generating functions of generalized logistic distribution based on lower $\mathrm{k}$ record values and its characterization. ProbStat Forum, 5, 47-53.

[11] Kumar, D. and Khan, M.I. (2012): Recurrence relations for moments of $\mathrm{k}$ th record values from generalized beta distribution and a characterization. Selçuk Journal of Applied Mathematics. 13, 75-82.

[12] Kumar, D., and Kulshrestha, A. (2013). Expectation Identities of Upper Record Values from Generalized Pareto Distribution and a Characterization. Journal of Statistics Applications \& Probability, 2 (2), 115-121.

[13] Lin, G.D. (1986). On a moment problem, Tohoku Math. J., 38, 595-598.

[14] Nain, K. (2010). Recurrence relations for single and product moments of $\mathrm{k}$ th record values from generalized Weibull distribution and a characterization. International Mathematical Forum, 5, 33, 1645-1652.

[15] Pawlas, P., and Szynal, D. (1999). Recurrence relations for single and product moment of $k$-th record values from Pareto, generalized Pareto and Burr distributions. Communication in Statistics-Theory and Methods, 28, 7, 1699-1709.

[16] Pawlas, P., and Szynal, D. (2000). Recurrence relations for single and product moment of $k$-th record values from Weibull distribution and a characterization, Journal of Applied Statistical Science, 10, 17- 25.

[17] Saran, J., and Singh, S.K. (2008). Recurrence relations for single and product moments of $\mathrm{k}$-th record values from linear exponential distribution and a characterization. Asian J. Math. Stat, 1, 159-164.

[18] Selim, M.A. (2011). Recurrence relations for single and product moments of $\mathrm{k}$-th record values from modified Weibull distribution and a characterization. Academy of Business Journal, AL-Azhar University, 8, 1, pp 75-87.

[19] Singh, S.K., Singh, U., and Sharma, V.K. (2013). Bayesian estimation and prediction for flexible Weibull model under type-II censoring scheme. Journal of Probability and Statistics, 2013. 